FACTA UNIVERSITATIS

Series: Mechanical Engineering Vol. 15, Nº 3, 2017, pp. 367 - 381

https://doi.org/10.22190/FUME171002020F

Original scientific paper

\title{
A NEW WINCH CONSTRUCTION FOR THE SMOOTH CABLE WINDING/UNWINDING
}

UDC 531

\author{
Mirjana Filipović ${ }^{1}$, Ljubinko Kevac ${ }^{2}$ \\ ${ }^{1}$ Mihajlo Pupin Institute, University of Belgrade, Serbia \\ ${ }^{2}$ School of Electrical Engineering, University of Belgrade, Serbia
}

\begin{abstract}
New constructive solutions of the winches for single-row radial multi-layered cable smooth winding/unwinding are described. Two new structural solutions of winches are defined. The nonlinear phenomenon of a cable smooth winding/unwinding process on the winch by using one of the two proposed constructive solutions is defined and analyzed. To facilitate understanding of this concept, the cable winding/unwinding process on only one winch is analyzed. The obtained variables which characterize the kinematics of the cable smooth winding/unwinding process are nonlinear and smooth. This result is important because the systems for the smooth cable winding/unwinding process on the winch could be parts of any cable driven mechanism. These systems can be used in various fields of human activity. For the verification of the presented theoretical contributions, a novel software package named SMOWIND - OW has been developed using MATLAB.
\end{abstract}

Key Words: Analysis, Kinematics, Cable Winding/unwinding, Winch Construction, Simulation

\section{INTRODUCTION}

The problem of the cable winding/unwinding on the winch is present in various systems used in different technical areas. These systems can have completely different applications and also different constructions but their characteristic is that they consist of one or more sub-systems for cable winding/unwinding. All these systems require stable control for implementation of defined task. Only several systems will be mentioned: measuring mechanism, machines in textile industry, cable logging systems in civil engineering and forestry, cranes, systems in shipping industry, CPR (Cable-suspended Parallel Robot) and other complex cable driven systems.

Received October 02, 2017 / Accepted November 22, 2017

Corresponding author: Mirjana Filipović

Mihajlo Pupin Institute, University of Belgrade, Volgina 15, 11000, Belgrade, Serbia

E-mail: mirjana.filipovic@pupin.rs 
For many decades, many researchers have dealt with analyzing the cable winding/ unwinding process on the winch. In this paper, only some of these investigations, namely, those that have inspired this research, will be presented. Authors of [1] have reviewed application of the cable logging systems. They have defined a user's manual which helps them to solve several problems such as protection of workers, soil and forests. Samset [2] has given a historic review of the systems which use winches for cable winding/ unwinding. The author gives information that these systems are used for more than five millennia which emphasizes their importance. Abdel-Rahman et al. [3] have analyzed dynamics and control of the cranes having cable winding/unwinding sub-systems as the main part. This is a review paper which shows historic development of cranes.

In [4-6], authors present theoretical and experimental contribution to analysis and synthesis of kinematics and dynamics of the winding/unwinding process of thread from a balloon. Fluctuating tensions in a perfectly flexible string unwinding from a stationary package is considered by Padfield [4]. Also, dependence of unwinding tensions on air resistance, unwinding speed, angle of winding on the package etc. is examined. Based on results of Padfield [4], Fraser et al. [5] have considered over-end unwinding of the yarn from a stationary helically wound cylindrical package. An improved theory for the variation of the yarn tension during high speed over-end unwinding from cylindrical yarn packages based upon the theory of the bent and twisted elastic rods is presented by Clark et al. [6].

Imanishi et al. [7] have presented a dynamic simulation of a wire rope involving both contacts with the winch drum and hydraulic systems using the finite element method. The rapid winch operation often causes a disorderly winding of the wire rope, which is an important quality problem. Dynamic simulation is, therefore, required for design of the hydraulic winch system on construction machinery. The wire rope is modeled using truss elements considering a large displacement motion.

Szczotka et al. [8] have presented the mathematical model of a pipelay spread. In the model, elasto-plastic deflections of the pipe, its large deformations and contact problems are considered. The modification of the rigid finite element method (REFM) is used to discretize the pipe.

Wire-guided control technologies are widely used to increase the targeting accuracy of advanced military weapons through the use of unwinding dispensers to guarantee that unwinding occurs without any problems, such as tangling or cutting. Lee et al. [9] have investigated transient behaviors of the cables unwinding from inner-winding cylindrical spool dispensers. The cable is withdrawn from the spool dispenser at a constant velocity through a fixed point located along the axis of the spool dispenser.

Filipovic et al. [10] have dealt with design, analysis and synthesis of the cable suspended parallel robotic system (CPR system). They have used a well-known winding/ unwinding sub-system presented by von Zietzwitz et al. [11].

A wide application of the process of cable winding/unwinding on the winch is important; that is why it deserves a detailed analysis and it is the subject of this paper. In this paper, two new forms of winches for ensuring single-row radial multi-layered cable smooth winding/unwinding have been constructed:

a) The first constructive solution is composed of two semicylindrical bodies of different radii: a two-cylinder winch.

b) The second constructive solution has a spiral shape: a spiral winch.

These two constructive solutions are the main contribution of this paper. 
For analysis and synthesis of the results from this paper a new software package SMOWIND - OW has been synthesized using MATLAB.

Kevac et al. [12] have presented a general form of mathematical model of the cable winding/unwinding system. Also, it can be noticed that the problem which will be analyzed in this paper is only one special case.

After Introduction, in Section 2 the basic theoretical principles of the kinematics of a smooth single-row radial multi-layered cable winding/unwinding process on the winch are presented. In Section 3 the program package SMOWIND - OW is defined. This program package is used to make simulation experiments and these simulations are shown in Section 4. Finally, the last part of the paper presents the conclusions, remarks, and plans for future research.

\section{THE BASIC THEORETICAL PRINCIPLES}

Kinematics of the single-row radial multi-layered smooth cable winding/unwinding process on the winch, hereinafter abbreviated as: the cable smooth winding/unwinding process on the winch, is a very complex and nonlinear process.

\subsection{Problem definition}

The solution presented in this paper comes as a result of analyzing the standard winch for single - row radial multilayered cable winding (unwinding) from Fig. 1. The analysis of the behavior of a circular winch intended for a single-row radial multi-layer cable winding/unwinding system indicates that this system is far from ideal and contains a series of constructive problems.

This simple construction of the winch has caused abrupt changes of important variables of this system: radius of cable winding/unwinding: $\mathrm{R}_{\mathrm{i}}$, cable length: $1 \mathrm{w}_{\mathrm{i}}$, and inclination of the cable with respect to $y_{i}$ axis: $\gamma_{i}$. A detailed description of this problem is given by Kevac et al. [13].

In this sub-section, the problem of cable winding/unwinding is only presented on standard winch for single - row radial multilayered cable winding (unwinding), but it should be emphasized that this problem is present in other forms of cable winding/unwinding systems, for example: a cable winding/unwinding system for a multi-row radial and axial cable winding/unwinding process, see Kevac et al. [12].

Because of the described effects, it is required to find a solution in the form of a new construction of the winch. Constructively, only a winding/unwinding system which does not generate abrupt changes of the variables involved presents a good system for this purpose.

The main point of this research is a geometric and mathematic definition of the cable winding/unwinding process on a novel shape of the winch intended for smooth rope (cable) winding/winding, unlike in the work by Kevac et al. [12], where the general form of mathematical model of the cable winding/unwinding system is defined.

\subsection{Problem solution}

In this paper, a kinematics model of the smooth cable winding/unwinding process on the winch will be developed. The new constructive solution of the winch is presented in Fig. 2 in which one can see a new shape of the winch which is adapted to the user's need that the 
winding/unwinding process should occur in a smooth and nonlinear fashion.
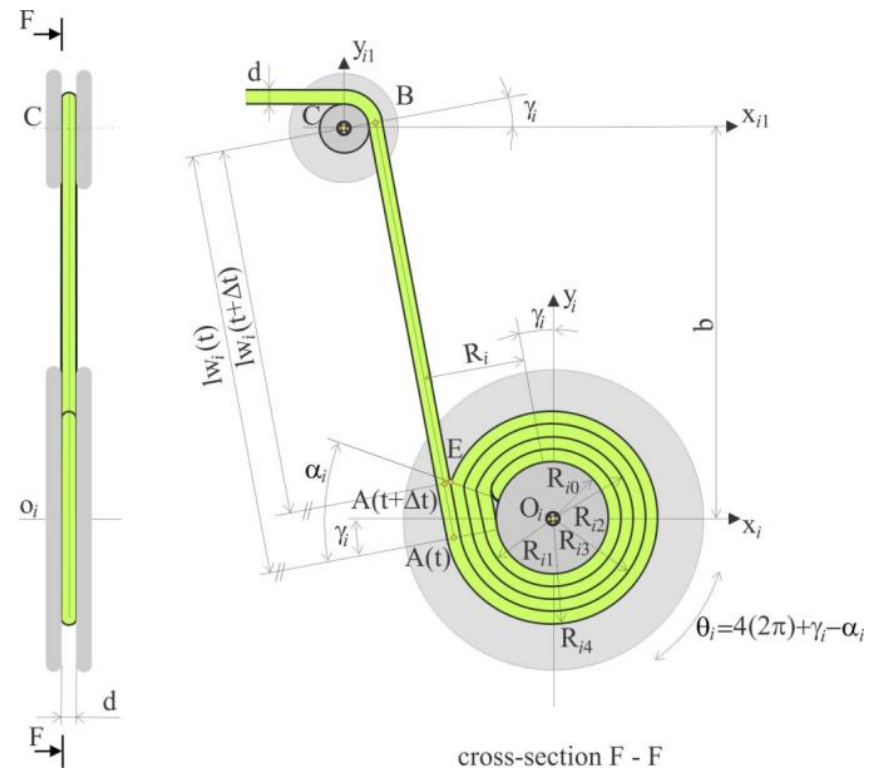

Fig. 1 Standard winch for the cable winding/unwinding system
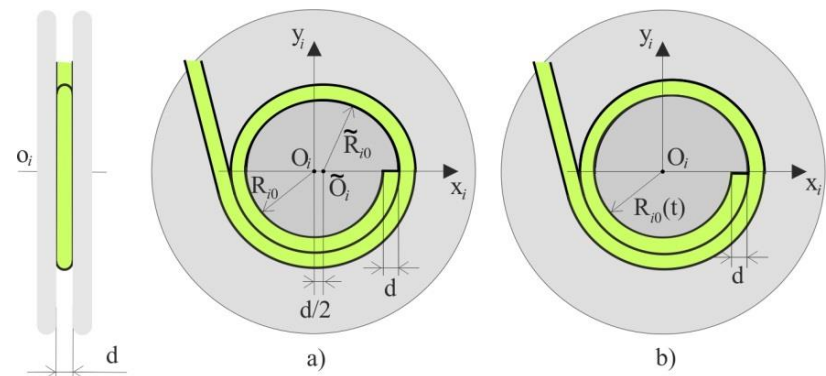

Fig. 2 Winch for the smooth cable winding/unwinding process:

a) two-cylinder winch, b) spiral winch

To achieve a smooth cable winding/unwinding process, two new constructive solutions of the winch are designed:

1) The first constructive solution consists of two semi cylindrical bodies of different radii, as shown in Fig. 2a. In view of the characteristics of this winch, it has been named a two-cylinder winch. The two-cylinder winch will be presented in this paper in detail, and

2) The second constructive solution has a spiral shape, as shown in Fig. 2b. It has been named a spiral winch.

Similar effects of the smooth cable winding/unwinding process are achieved for both the spiral and the two-cylinder winch. 
In this paper, the two - cylinder winch will be used for performing the corresponding theoretical analysis. In the continuation of this Section, the geometry of the cable smooth winding/unwinding process on one winch will be shown and analyzed in detail. The cable is mounted so that it emerges from the winch at a place where there is a joint of two semi cylindrical bodies.

The starting position of the cable during the winding/unwinding process on the winch is shown in Fig. 3. The starting position is systematically (by calibration) defined to be at the direction of the negative part of $x_{i}$ axis. Selection of the starting position affects further kinematics of the cable winding/unwinding process on the winch.

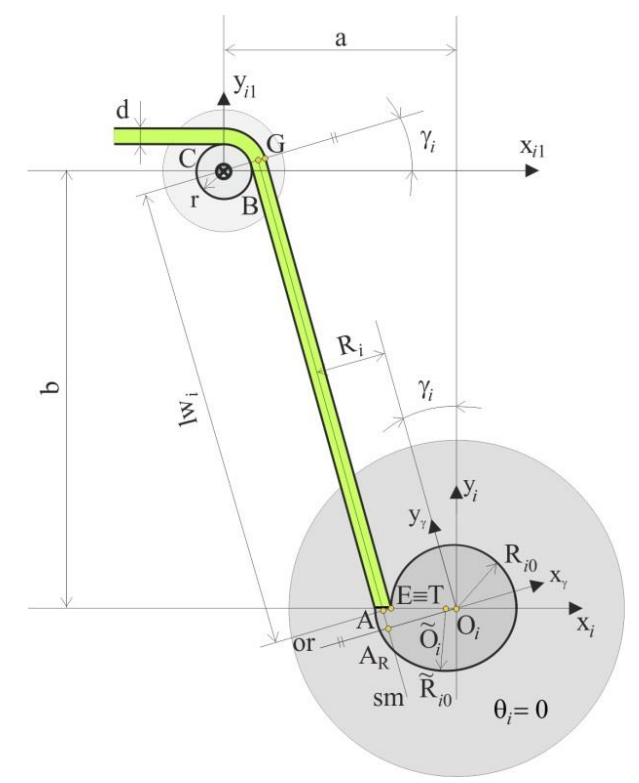

Fig. 3 The starting position of the winding/unwinding system

A more detailed look at the starting position of the system for cable winding/unwinding is shown in Fig. 4a. In order to facilitate understanding of the kinematics of the cable smooth winding/unwinding process on the winch, the geometry of this complex process will be shown.

Defining the geometry of the cable smooth winding/unwinding process is needed for specifying the kinematic and dynamic models of this process. Also, for a good understanding it is sufficient only to analyze the winding process of the cable on the winch; thus the analysis of the process of unwinding will be omitted since the phenomena are the same but only occur in the reverse order with respect to winding.

Figure 3 shows the starting position of the system for the smooth winding/unwinding of the cable on the winch. This system consists of a newly shaped motorized winch, hereinafter referred to as winch, which is positioned along one axis of the $x_{i}-y_{i}$ coordinate system. This winch (winds up)/unwinds a cable of diameter $\mathrm{d}=0.0008 \mathrm{~m}$. From the other side this cable goes over a smaller pulley (which is not motorized) which has radius $r=0.009 \mathrm{~m}$. This pulley is positioned at the center of $x_{i 1}-y_{i 1}$ coordinate system. The axis of rotation of the pulley is 
positioned at the base of this coordinate system, point $C$. This point in relation to the $\mathrm{x}_{\mathrm{i}}-\mathrm{y}_{\mathrm{i}}$ coordinate system has coordinates $(-a, b)$. Distance $a=0.045 \mathrm{~m}$ presents a horizontal distance between rotation axes of the winch and the pulley, while distance $b=0.524 \mathrm{~m}$ presents a vertical distance between these two axes.

The new shape of the winch is composed of two semicylindrical bodies. The smaller semicylinder has a basis of radius $\mathrm{R}_{\mathrm{i} 0}=0.0136 \mathrm{~m}$ with the center at point $\mathrm{O}_{\mathrm{i}}(0,0)$. Radius of the bigger semicylinder $\tilde{R}_{i 0}$ is geometrically defined by the shape function of this winch in relation to the radius of the smaller semicylinder $\mathrm{R}_{\mathrm{i} 0}$. The bigger semicylinder has a radius of basis $\tilde{\mathrm{R}}_{\mathrm{i} 0}=\mathrm{R}_{\mathrm{i} 0}+\mathrm{d} / 2=0.014 \mathrm{~m}$ with the center at point $\widetilde{\mathrm{O}}_{\mathrm{i}}(-\mathrm{d} / 2,0)$.

The system for smooth winding/unwinding of the cable on the winch is constructed so that when shown in the plane perpendicular to the winding/unwinding axis, it looks as shown in Fig. 3. To simplify the terminology, the two semicylinders will further on be referred to as two semicircles.

The center of the smaller semicircle is at the origin, $\mathrm{O}_{\mathrm{i}}$, of the coordinate system $x_{i}-y_{i}$. The rotation axis of the motorized winch is labeled as $\mathrm{o}_{\mathrm{i}}$. Unlike point $\mathrm{O}_{\mathrm{i}}$, the center of the bigger semicircle, point $\widetilde{\mathrm{O}}_{\mathrm{i}}$, keeps on changing its position constantly during the cable winding/unwinding process on the winch. Point $\widetilde{\mathrm{O}}_{\mathrm{i}}$ is constantly rotating around point $\mathrm{O}_{\mathrm{i}}$ at distance $\mathrm{d} / 2$.

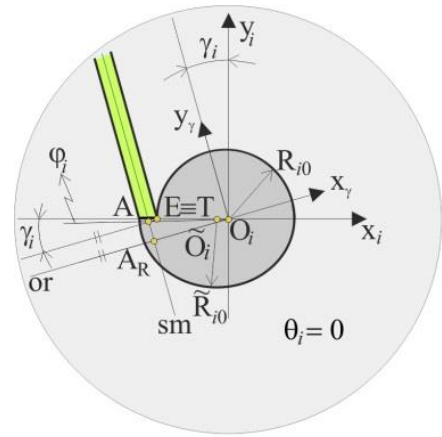

a)

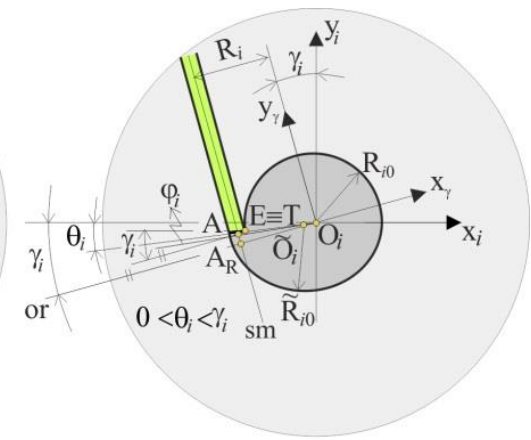

b)

Fig. 4 a) The starting position of the winding/unwinding system - close-up;

b) Position of the winding/unwinding system for $0<\theta<\gamma_{i}$

A better view of the winch is shown in Fig. 4a. Here one can clearly see the place where the cable emerges from the winch. Point E belongs to the outer contour of the winch and it is positioned at the intersection of the smaller semicircle and the flat part of the winch. At the starting position, this point belongs to the negative part of $x_{i}$ axis. This point has a stationary position in comparison with the winch during the whole of the cable winding/unwinding process on the winch. It is assumed that the deflection angle between line $\mathrm{O}_{\mathrm{i}} \mathrm{E}$ and negative part of $\mathrm{x}_{\mathrm{i}}$ axis defines the winding/unwinding process of the cable. This deflection is denoted as angle $\theta_{\mathrm{i}}$ whose value at the starting position is:

$$
\theta_{\mathrm{i}}=0
$$


Unlike point $\mathrm{E}$, point $\mathrm{T}$ constantly changes its position on the winch. This point presents the place where the cable touches the winch or the cable wound so far. At the starting position, points $\mathrm{E}$ and $\mathrm{T}$ overlap, as can be seen in Figs. 3 and $4 \mathrm{a}$. The winch and the pulley are positioned so that angle $\gamma_{\mathrm{i}}$ has a positive value at any moment. Angle $\gamma_{\mathrm{I}}$ presents one of the variables of the system which characterize the cable winding/unwinding process. At the starting position, angle $\gamma_{\mathrm{i}}$ has the largest value of $0.04116 \mathrm{rad}$, which is defined by the constructive solution of the mechanical part. This angle is defined in the following fashion: the first leg of the angle is the line running through origin $\mathrm{O}_{\mathrm{i}}$ in parallel with the tangent drawn at point $\mathrm{T}$ to the circle having radius $\mathrm{r}+\mathrm{d}$, centered at point $\mathrm{C}$; the second leg is positive part of the $\mathrm{y}_{\mathrm{i}}$ axis.

It is presumed that the cable is wound at constant angular speed, i.e. $\dot{\theta}_{i}=$ const. This presumption presents an idealized theoretical condition, which has been introduced for an easier and clearer explanation of the smooth cable winding process on the winch.

It is assumed that the cable force acts through the central axis of the cable, i.e. along direction of the line sm (see Figs. 3 and 4a). Usually, it is presumed that the winding/ unwinding radius is calculated at the position where the force acts upon the winch. With this presumption and from Figs. 3 and $4 \mathrm{a}$ it can be seen that this radius presents distance $\mathrm{O}_{\mathrm{i}} \mathrm{A}_{\mathrm{R}}$ at the starting position. The value of this radius can be calculated as follows:

$$
\mathrm{R}_{\mathrm{i}}=\mathrm{O}_{\mathrm{i}} \mathrm{A} \cdot \cos \left(\gamma_{\mathrm{i}}-\varphi_{\mathrm{i}}\right)
$$

At the starting position, point $\mathrm{A}$ is positioned at the intersection between line $\mathrm{O}_{\mathrm{i}} \mathrm{A}$ and the line which contains point $\mathrm{T}$ and is rotated about point $\mathrm{T}$ by angle $\gamma_{\mathrm{i}}$.

The angle between lines $\mathrm{O}_{\mathrm{i}} \mathrm{A}$ and $\mathrm{O}_{\mathrm{i}} \mathrm{T}$ is labeled as $\varphi_{\mathrm{i}}$. This angle has the biggest value at the starting position. The position of point $A_{R}$ is at the intersection between lines sm and or. Point $\mathrm{B}$ is positioned at the intersection between line sm and the line which contains point $\mathrm{C}$ and which is parallel to line or. Distance $A B$ labeled as $l_{\mathrm{i}}$ presents a dynamic variable during the cable smooth winding/unwinding process on the winch. This variable has its dynamics of change and thus affects the dynamic response of the system (see Fig. 3).

Figure $4 \mathrm{~b}$ shows the position of the system when values of angle $\theta_{\mathrm{i}}$ meet the following requirement:

$$
0<\theta_{\mathrm{i}}<\gamma_{\mathrm{i}}
$$

For this position of the system, the winding/unwinding radius is calculated as:

$$
\mathrm{R}_{\mathrm{i}}=\mathrm{O}_{\mathrm{i}} \mathrm{A} \cdot \cos \left(\gamma_{\mathrm{i}}-\varphi_{\mathrm{i}}-\theta_{\mathrm{i}}\right)
$$

As in the previous case (Figs. 3 and $4 a$ ), point $A_{R}$ is positioned at the intersection of lines sm and or, while point $\mathrm{A}$ is at the intersection of line sm and the line which contains point $\mathrm{T}$ and is parallel to line or. In relation to the initial value of angle $\gamma_{\mathrm{i}}$, its value is smaller now, while distance $1 w_{i}$ is growing in relation to its initial value. The angle between lines $\mathrm{O}_{\mathrm{i}} \mathrm{A}$ and $\mathrm{O}_{\mathrm{i}} \mathrm{T}$ decreases during the period of winding which is specified by Eq. (3). The system's position which is analyzed next is shown in Fig. 5a. This is the position when the following condition is satisfied:

$$
\theta_{\mathrm{i}}=\gamma_{\mathrm{i}}
$$

At this moment, points $A_{R}$ and $A$ are at the same position and they are lying on line $\mathrm{O}_{\mathrm{i}} \mathrm{T}$. From this moment on, point $\mathrm{A}$ is always on line $\mathrm{O}_{\mathrm{i}} \mathrm{T}$ during the winding process. At this 
moment, angle $\varphi_{\mathrm{i}}$ achieves value 0 and further on it does not affect the cable winding process. In Fig. 5a, it can be seen that line $\mathrm{O}_{\mathrm{i}} \mathrm{T}$ is lying on $\mathrm{x}_{\gamma}$ axis (also line $\mathrm{O}_{\mathrm{i}} \mathrm{E}$ is lying on this axis) - axis $x_{\gamma}$ presents the axis that is deflected by angle $\gamma_{i}$ compared to the positive part of $x_{i}$ axis. Coordinate system $x_{\gamma}-y_{\gamma}$ is always defined by angle $\gamma_{i}$. At this moment, the cable is tangential to the winch at point $\mathrm{T}$. Also, it is the last moment when points $\mathrm{E}$ and $\mathrm{T}$ overlap. From that moment on, point $\mathrm{E}$ maintains its fixed position on the body of the winch, while point $\mathrm{T}$ follows winding dynamics of the cable.

At this moment, the winding/unwinding radius has the following value:

$$
\mathrm{R}_{\mathrm{i}}=\mathrm{O}_{\mathrm{i}} \mathrm{A}=\mathrm{O}_{\mathrm{i}} \mathrm{A}_{\mathrm{R}}=\mathrm{R}_{\mathrm{i} 0}+\mathrm{d} / 2
$$

Also, at this moment, distance $1 \mathrm{w}_{\mathrm{i}}$ has the biggest value during the cable winding process on the winch.

The cable keeps on winding and angle $\theta_{\mathrm{i}}$ takes values from the following range:

$$
\gamma_{\mathrm{i}}<\theta_{\mathrm{i}}<\pi+\gamma_{\mathrm{i}}
$$

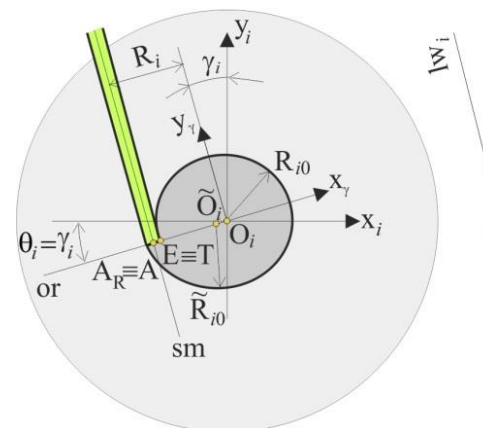

a)

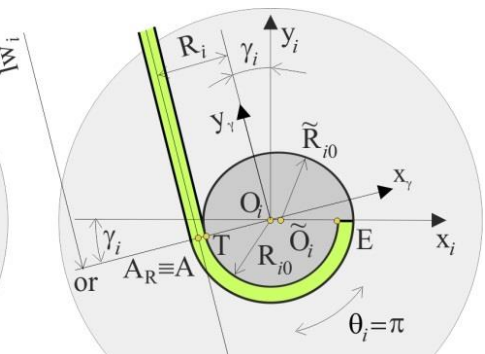

$\mathrm{sm}$

b)

Fig. 5 Position of the system for a) $\theta_{i}=\gamma_{i}$ and b) $\theta_{i}=\pi$

The range defined by Eq. (7) has been named a con range (constant). Throughout this range, radius $R_{i}$, length $1 w_{i}$, and angle $\gamma_{i}$ (important variables of the cable smooth winding/ unwinding process) have constant values which they have acquired at the moment when the condition defined by Eq. (5) is satisfied. An example of the position from this range is shown in Fig. 5b. In this example line $\mathrm{O}_{\mathrm{i}} \mathrm{E}$ overlaps with the positive part of $\mathrm{x}_{\mathrm{i}}$ axis, i.e. $\theta_{i}=\pi$. From Fig. $5 b$ it can be seen that positions of points $\mathrm{E}$ and $\mathrm{T}$ are different.

An important moment is when the following condition is satisfied:

$$
\theta_{\mathrm{i}}=\pi+\gamma_{\mathrm{i}}
$$

because at that moment the system leaves the con range and a new law of change of all relevant variables: winding/unwinding radius $R_{i}$ length $1 w_{i}$, and angle $\gamma_{i}$ starts. It can be seen that at this moment line $\mathrm{O}_{\mathrm{i}} \mathrm{E}$ lies on $\mathrm{x}_{\gamma}$ axis (see Fig. 6a), but, in comparison with the positions from Fig. 5a, this line is rotated about point $\mathrm{O}_{\mathrm{i}}$ by angle $\pi$. After that moment, the cable is starting to wind smoothly on the part of the winch with a bigger radius, i.e. a bigger semicircle. 
From that moment on, angle $\theta_{\mathrm{i}}$ takes the values defined by the following equation:

$$
\pi+\gamma_{\mathrm{i}}<\theta_{\mathrm{i}}<2 \pi+\gamma_{\mathrm{i}}
$$

The range when all the relevant variables are changing their values is named a smvar (smooth variable) and one position of the system within this range is shown in Fig. 6b.

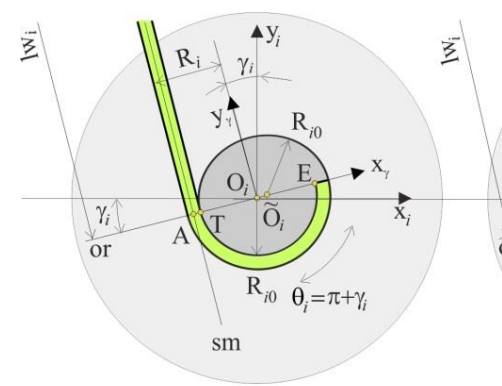

a)

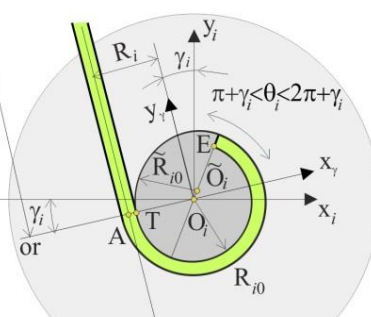

sm

Fig. 6 Position of the system for: a) $\theta_{i}=\pi+\gamma_{i}$ and b) $\pi+\gamma_{i}<\theta_{i}<2 \pi+\gamma_{i}$
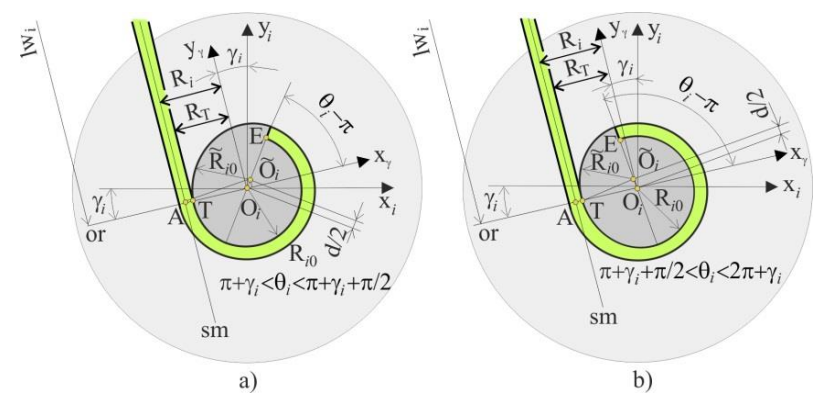

b)

Fig. 7 Position of the system for: a) $\pi+\gamma_{i}<\theta_{i}<\pi+\gamma_{i}+\pi / 2$, and b) $\pi+\gamma_{i}+\pi / 2<\theta_{i}<2 \pi+\gamma_{i}$

Angle $\theta_{\mathrm{i}}$ is changing linearly during the cable winding process, i.e. angular speed of the winch rotation is constant, $\dot{\theta}_{\mathrm{i}}=$ const. The defined smvar range represents the period when cable winding/unwinding radius $R_{i}$ changes its value. Upon entering this range, during the cable winding, this radius starts to grow from value $R_{i}=R_{i 0}+d / 2$ to value $R_{i}=$ $\mathrm{R}_{\mathrm{i} 0}+3(\mathrm{~d} / 2)$ which is reached at the end of the smvar range. For an easier description of the change of radius $R_{i}$, two sub-ranges of the smvar range will be considered:

1) The first sub-range is defined by the following change of angle $\theta_{\mathrm{i}}: \pi+\gamma_{\mathrm{i}}<\theta_{\mathrm{i}}<\pi+\gamma_{\mathrm{i}}$ $+\pi / 2$. This period of winding is shown in Fig. 7a and in this sub-range of the smvar range, radius $R_{i}$ is calculated by $R_{i}=R_{T}+d / 2$. Radius $R_{T}$ becomes a changing variable in the smvar range, where at the beginning of the range it has value $R_{T}=R_{i 0}$. During the first sub-range, the change of this variable is described by the following Eq.:

$$
\mathrm{R}_{\mathrm{T}}=\sqrt{\left(\mathrm{R}_{\mathrm{i} 0}+\mathrm{d} / 2\right)^{2}-\left(\mathrm{d} / 2 \cdot \sin \left(\theta_{\mathrm{i}}-\pi\right)\right)^{2}}-\mathrm{d} / 2 \cdot \cos \left(\theta_{\mathrm{i}}-\pi\right)
$$


2) The second sub-range is defined by the following change of angle $\theta_{\mathrm{i}}: \pi+\gamma_{\mathrm{i}}+\pi / 2<\theta_{\mathrm{i}}<$ $2 \pi+\gamma_{\mathrm{i}}$. This period is shown in Fig. $7 \mathrm{~b}$ and in this sub-range of the smvar range, radius $\mathbf{R}_{\mathrm{i}}$ is calculated by $R_{i}=R_{T}+d / 2$, where radius $R_{T}$ is:

$$
\mathrm{R}_{\mathrm{T}}=\sqrt{\left(\mathrm{R}_{\mathrm{i} 0}+\mathrm{d} / 2\right)^{2}-\left(\mathrm{d} / 2 \cdot \sin \left(\theta_{\mathrm{i}}-\pi\right)\right)^{2}}+\left|\mathrm{d} / 2 \cdot \cos \left(\theta_{\mathrm{i}}-\pi\right)\right|
$$

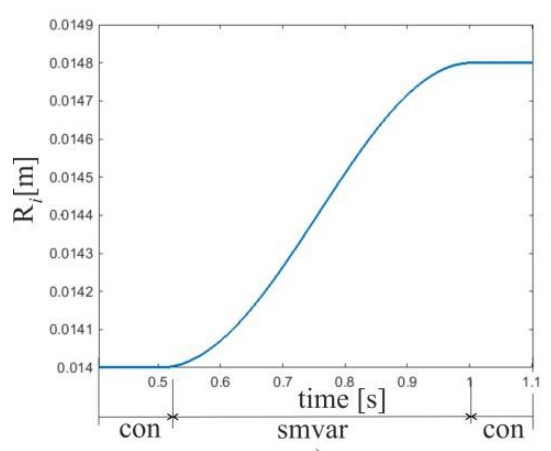

a)

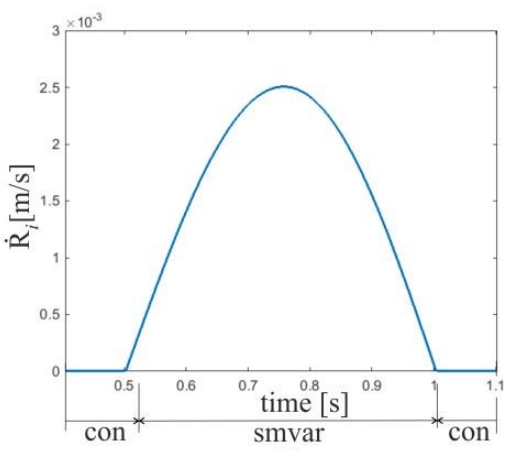

b)

Fig. 8 a) Radius $R_{i}$ and b) the first derivative of radius $R_{i}$ over the smvar range

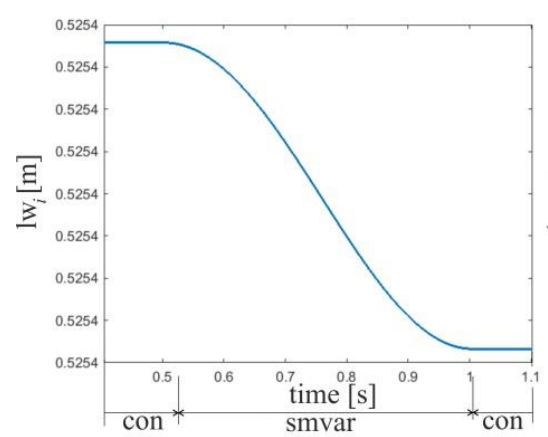

a)

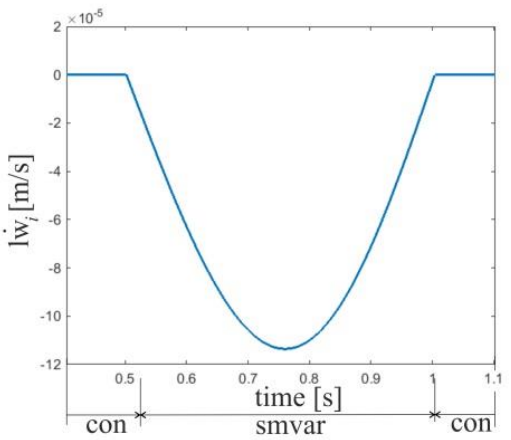

b)

Fig. 9 a) Length $l w_{i}$ and b) the first derivative of length $l w_{i}$ over the smvar range

Change of the winding/unwinding radius achieved in this fashion is smooth, as can be seen from Fig. $8 \mathrm{a}$, where variation of radius $\mathrm{R}_{\mathrm{i}}$ over the smvar region is presented. Figure $8 \mathrm{~b}$ shows the first derivative of this radius, i.e. variable $\dot{\mathrm{R}}_{\mathrm{i}}$, where one can see a smooth change of the winding/unwinding radius. It can be seen that during the cable winding process the radius is slowly growing towards the already defined value of $R_{i}=R_{i 0}+3(d / 2)$.

Upon entering the smvar range, points $\mathrm{T}$ and $\mathrm{A}$ are slowly changing their positions. Because of that distance $1 \mathrm{w}_{\mathrm{i}}$ gradually decreases in its value during this period of winding. This phenomenon can be seen in Fig. 9a, where variation of distance ${ }^{1} w_{i}$ over the smvar range is presented. This change has a similar dynamics like radius $\mathrm{R}$, only it is decreasing during the cable winding process. Figure $9 \mathrm{~b}$ shows the change of the first derivative of this variable, $l \dot{\mathrm{w}}_{\mathrm{i}}$. 


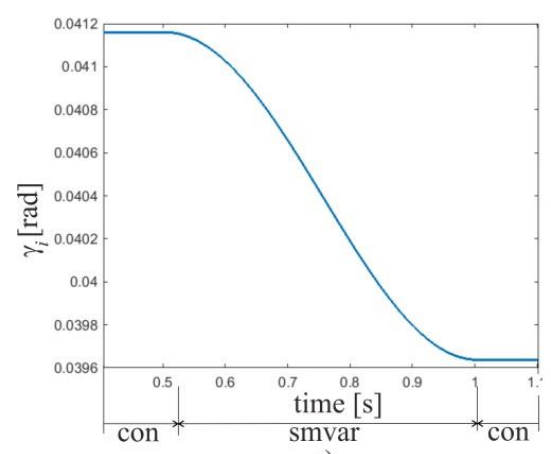

a)

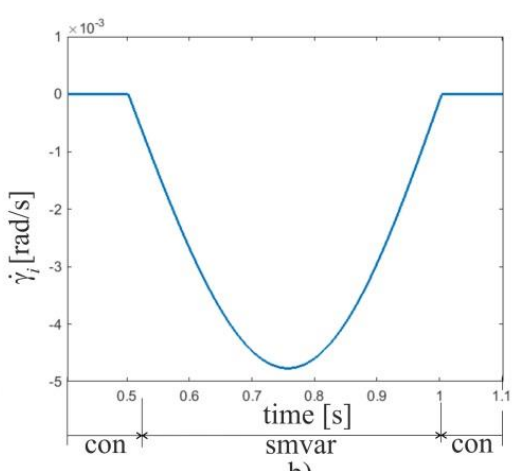

b)

Fig. 10 a) Angle $\gamma_{\imath}$ and b) the first derivative of angle $\gamma_{\imath}$ over the smvar range

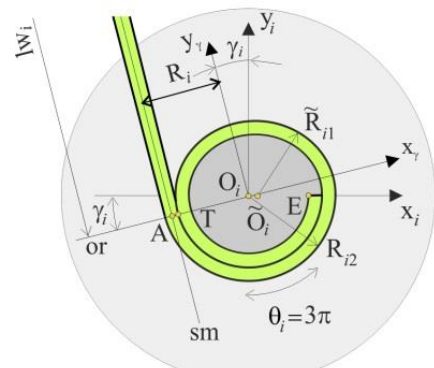

a)

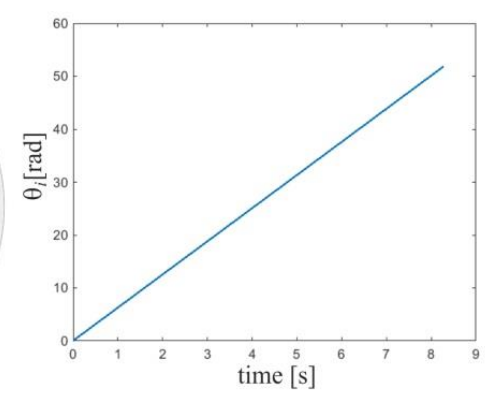

b)

Fig. 11 a) Position of the system for $\theta_{i}=3 \pi$ and b) angle $\theta_{i}$ : $0<\theta_{i}<17 \pi$

Because of the change of positions of points $\mathrm{T}$ and $\mathrm{A}$, angle $\gamma_{\mathrm{I}}$ is decreasing in the smvar range. The change of this angle over the smvar range is shown in Fig. 10a, while Fig. 10b shows the first derivative of this variable, $\dot{\gamma}_{i}$.

At the moment when angle $\theta_{\mathrm{i}}$ reaches the following value:

$$
\theta_{\mathrm{i}}=2 \pi+\gamma_{\mathrm{i}}
$$

the system goes out of the smvar range and enters a new con range. At this moment, winding/unwinding radius $\mathrm{R}_{\mathrm{i}}$ has the following value:

$$
\mathrm{R}_{\mathrm{i}}=\mathrm{R}_{\mathrm{i} 0}+3 \cdot \mathrm{d} / 2
$$

From this moment on, the system for smooth cable winding/unwinding on the winch enters the period when angle $\theta_{\mathrm{i}}$ has values defined by:

$$
2 \pi+\gamma_{\mathrm{i}}<\theta_{\mathrm{i}}<3 \pi+\gamma_{\mathrm{i}}
$$

Winding/unwinding radius $R_{i}$, length $\mathrm{lw}_{\mathrm{i}}$, and angle $\gamma_{\mathrm{i}}$ have constant values over the new con range and they keep the values achieved when angle $\theta_{i}$ had value of $2 \pi+\gamma_{i}$, see Eq. (12). Figure 11a shows one example of the system during the new con range defined by Eq. (14): position of the system for $\theta_{\mathrm{i}}=3 \pi$. 
Based on the process defined in this Section, it can be concluded that the smooth cable winding/unwinding process on the winch is accomplished by a cyclical alternation of the con and smvar ranges.

\section{THE PROGRAM PACKAGE SMOWIND - OW}

Based on mathematical Eqs. (1-14), a program package SMOWIND - OW (SMOoth Winding for One Winch) has been synthesized in MATLAB. This program package includes definition of the motion dynamics of only one two-cylinder winch for the cable smooth winding/unwinding process. A smooth trajectory of the winch is defined for the overall range of angle $\theta_{\mathrm{i}}: 0<\theta_{\mathrm{i}}<17 \pi \mathrm{rad}$. It is presumed that angular speed of this winch is constant. The program is generated so that the motion takes place only in the direction of the cable winding on the winch. It is assumed that the cable unwinding takes place in the same way but in the opposite direction. This program package gives an ability to the user to track the change of dynamics of all the relevant variables, e.g. winding/unwinding radius, length between the winch and hanging point, etc. The program package presumes application of a two-cylinder winch (Fig. 2a), but with small modifications this program package can be used for generation of the same results for the spiral winch (Fig. 2b), if required. By using this program package, the user can track changes of the first derivatives of all the relevant variables during any part of the winding process within the defined overall range of angle $\theta_{\mathrm{i}}$. The cyclical character of the winding process is shown in detail in the next Section where the simulation experiments performed by the program package SMOWIND - OW are presented.

\section{CyClicality of THE PROCESS}

In Section 2, the basic principles of kinematics of the smooth cable winding on one two-cylinder winch, shown in Fig. 2a, are presented. The cable winding process has been shown for the values of angle $\theta_{\mathrm{i}}$ given by following inequality: $0<\theta_{\mathrm{i}}<3 \pi+\gamma_{\mathrm{i}}$, see Fig. 11a.

Based on this analysis, it is concluded that a smooth cable winding process involves cyclical alterations of the con and smvar ranges. With a constant growth of angle $\theta_{i}$, i.e. with a continuous cable winding, the previously defined principles of winding should be applied to achieve a smooth growth of radius $\mathrm{R}_{\mathrm{i}}$ and a smooth decrease of angle $\gamma_{\mathrm{i}}$ and length $1 \mathrm{w}_{\mathrm{i}}$. By using the program package SMOWIND - OW, which was outlined in Section 3, the simulation experiments for a linear full scale increase of angle $\theta_{\mathrm{i}}, 0<\theta_{\mathrm{i}}<17 \pi$, have been generated. The change of angle $\theta_{\mathrm{i}}$ is shown in Fig. 11b. From this figure it can be seen that this angle has a linear change, i.e. its first derivative is constant $\dot{\theta}_{\mathrm{i}}=$ const (the angular speed of the winch is constant). Figure $12 \mathrm{a}$ shows the change of radius $\mathrm{R}_{\mathrm{i}}$ for this period of winding. During the winding period, a growth of radius $R_{i}$ has a cyclical character. It can be noticed that radius $R_{i}$ has a constant and gradual growth in smvar ranges, while it has a constant value during con ranges. Figure $12 \mathrm{~b}$ shows the first derivative of variable $\mathrm{R}_{\mathrm{i}}$. From this figure it can be seen that the first derivative of radius $R_{i}$ has a smooth and cyclical change with maximal value of $\dot{\mathrm{R}}_{\mathrm{i} \text { max }}=0.0025 \mathrm{~m} / \mathrm{s}$. 


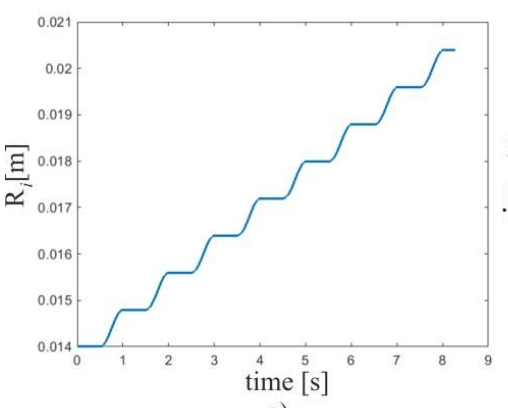

a)

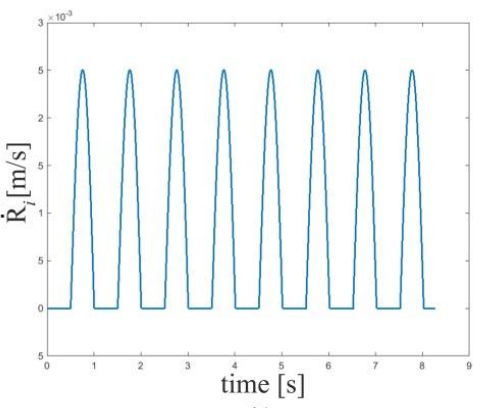

b)

Fig. 12 a) Radius $R_{i}$, and b) the first derivative of radius $R_{i}$

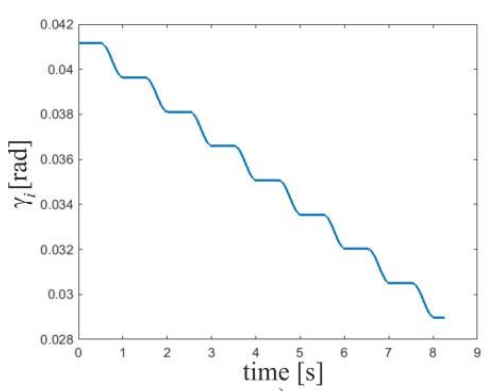

a)

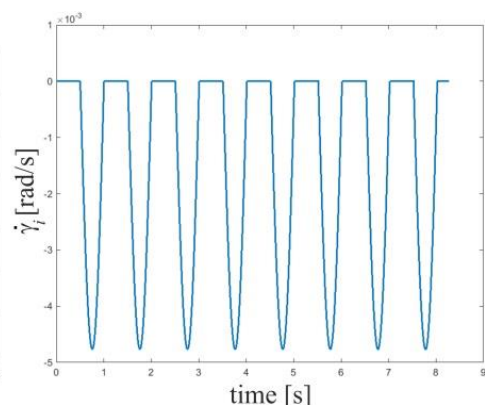

b)

Fig. 13 a) Angle $\gamma_{i}$ and b) the first derivative of angle $\gamma_{i}$

Angle $\gamma_{\mathrm{i}}$ is decreasing during the cable winding process on the winch. The change of this variable is similar to the change of the winding/unwinding radius, as can be seen in Fig. 13a. Also, Fig. 13b shows the first derivative of this variable. It can be seen that its maximum absolute value is $\dot{\gamma}_{\mathrm{imax}}=0.0047 \mathrm{rad} / \mathrm{s}$.

Length $\mathrm{lw}_{\mathrm{i}}$ is also decreasing during the cable winding process and the character of its change is almost the same as the character of the change of angle $\gamma_{i}$, as can be seen in Fig. 14a. Figure 14b shows the change of its first derivative and it can be seen that its maximum absolute value is $1 \dot{\mathrm{w}}_{\mathrm{i} \operatorname{mx}}=1.4 \cdot 10^{-4} \mathrm{~m} / \mathrm{s}$.

The change of the cable movement velocity is much smaller than that achieved with the standard winch form, see Kevac et al. [13]. This presents only one advantage of the novel winch in comparison with the standard one. What is essentially important about this new constructive solution of the winch is that abrupt changes of the cable length velocity are avoided.

It can be seen that first derivatives of all important variables $\dot{\mathrm{R}}_{\mathrm{i}}, 1 \dot{\mathrm{w}}_{\mathrm{i}}, \dot{\gamma}_{\mathrm{i}}$ have small smooth changes and thus their effect on the whole system's dynamics is much better in terms of the systems' dynamic response.

The figures shown in this Section (Figs. 11b-14) correspond to the idealized case of the cable winding process on a two-cylinder winch. Also, in these figures only winding of one cable on one winch under ideal conditions, when the angular speed of the winch is constant, $\dot{\theta}_{\mathrm{i}}=$ const, is analyzed. 

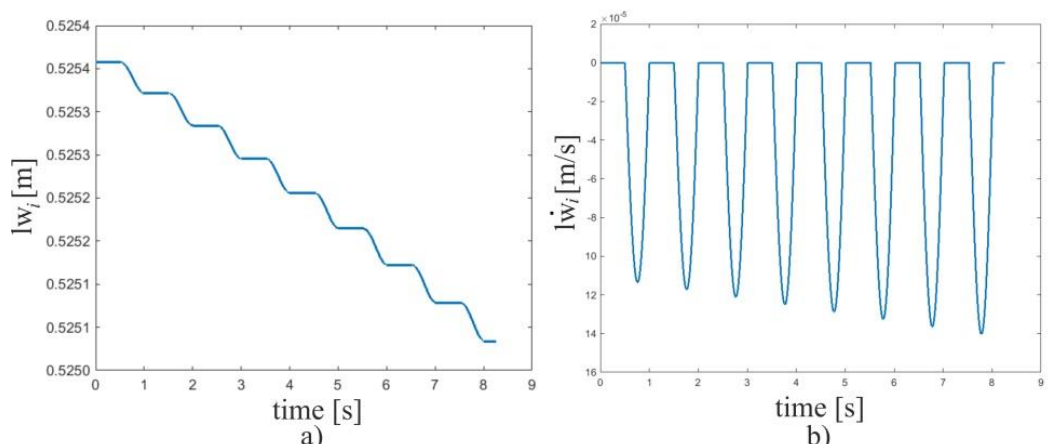

Fig. 14 a) Length $l w_{i}$ and $\left.b\right)$ the first derivative of length $l w_{i}$

\section{CONCLUSIONS}

The concept of the nonlinear smooth cable winding/unwinding process on the winch has been defined and analyzed. This presents a novelty in the literature published so far. It has been developed due to our intention to define a new form of winches for a single-row radial multi-layered smooth cable winding/unwinding process. Two novel mechanical constructions of the winch have been defined: one named a two-cylinder winch and the other named a spiral winch. The two-cylinder winch has been used for further kinematics investigations. To explain the idea, the process of the smooth cable winding/unwinding on one two-cylinder winch has been analyzed and modeled. A detailed synthesis and analysis are performed of the winding process only since it is assumed that the process of unwinding is identical except for its flowing in the reverse direction. Idealized conditions during the winding process have been assumed, i.e. it is presumed that the winch is always rotating with a constant angular speed. Under these conditions, it is discovered that the smooth cable winding/unwinding process has two ranges: a range smvar when all the relevant variables change their values: winding/unwinding radius $R_{i}$, angle $\gamma_{i}$, and length $1 w_{i}$ and a con range when these variables have constant values. For the verification and validation of the defined theoretical concept, a novel program package SMOWIND - OW has been developed using MATLAB. By using this program package, the simulation experiments of smooth winding of the cable on one winch have been made and the presented figures show the changes of: radius $R_{i}$, angle $\gamma_{i}$, length $l w_{i}$ and their first derivatives. From these figures it can be seen that these variables have smooth changes during rotation of the winch.

Future research in this area would concern the inclusion of the proposed new construction of the winch in a complex cable suspended system, which may consist of $n$ subsystems performing cable winding/unwinding process such in Aref et al. [14].

Acknowledgements: This research was supported by the Ministry of Education, Science and Technological Development, Government of the Republic of Serbia through the following two projects: Grant TR-35003, "Ambientally intelligent service robots of anthropomorphic characteristics", by Mihajlo Pupin Institute, University of Belgrade, Serbia, and Grant OI-174001, "The dynamics of hybrid systems of complex structure", by the SANU Institute Belgrade and Faculty of Mechanical Engineering University of Nis, Serbia. We are grateful to Prof. Dr. Katica R. (Stevanovic) Hedrih from the 
Mathematical Institute, Belgrade for the helpful consultations during this research and we are grateful to our former colleague Zivko Stikic for his help during this research.

\section{REFERENCES}

1. United Nations, 1981, Cable Logging Systems, FOOD AND AGRICULTURE ORGANIZATION OF THE UNITED NATIONS, Roma.

2. Samset, I., 1985, Winch and cable systems, Series Forestry Sciences, Vol. 18, Springer Netherlands.

3. Abdel-Rahman, E.M., Nayfeh, A.H., Masoud, Z.N., 2003, Dynamics and Control of Cranes: A Review, Journal of Vibration and Control, 9, pp. 863-908.

4. Padfield, D.G., 1958, The motion and tension of an unwinding thread. I, Proc. R. Soc. Lond. A, 245(1242), pp. 382-407.

5. Fraser, W.B., Ghosh, T.K., Batra, S.K., 1992, On Unwinding Yarn from a Cylindrical Package, Proc. R. Soc. Lond. A, 436(1898), pp. 479-498.

6. Clark, J.D., Fraser, W.B., Stump, D.M., 2001, Modelling of tension in yarn package unwinding, Journal of Engineering Mathematics, 40, pp. 59-75.

7. Imanishi, E., Nanjo, T., Kobayashi, T., 2009, Dynamic simulation of wire rope with contact, Journal of Mechanical Science and Technology, 23, pp. 1083-1088.

8. Szczotka, M., Wojciech, S., Maczynski, A., 2007, Mathematical model of a pipelay spread, The Archive of Mechanical Engineering, 54(1), pp. 27-46.

9. Lee, J-W., Kim, K-W., Kim, H-R., Yoo, W-S., 2012, Prediction of unwinding behaviors and problems of cables from inner-winding spool dispensers, Nonlinear Dyn., 67, pp.1791-1809.

10. Filipovic, M., Djuric, A., Kevac, Lj., 2015, The significance of adopted Lagrange principle of virtual work used for modeling aerial robots, Applied Mathematical Modelling, 39(7), pp. 1804-1822.

11. von Zietzwitz, J., Fehlberg, L., Bruckmann, T., Vallery, H., 2013, Use of passively guided deflection units and energy-storing elements to increase the application range of wire robots, in Bruckmann, T., Pott, A. (Eds.), Cable-Driven Parallel Robots, Mechanisms and Machine Science, vol. 12, pp. 167-184.

12. Kevac, Lj., Filipovic, M., 2017, Mathematical Model of Cable Winding/Unwinding System, Journal of Mechanics, DOI: https://doi.org/10.1017/jmech.2017.59,

13. Kevac, Lj., Filipovic, M., Rakic, A., 2017, Dynamics of the process of the cable winding (unwinding) on the winch, Applied Mathematical Modelling 48, pp. 821-843.

14. Aref, M.M., Taghirad, H.D., 2008, Geometrical workspace analysis of a cable-driven redundant parallel manipulator: KNTU CDRPM. Proc. IEEE/RSJ International Conference on Intelligent Robots and Systems (IROS), pp. 1958-1963. 